SLAC-PUB-7806

CERN-TH/98-362

thep-ph $[9811325$

November 1998

\title{
Illuminating dark matter and primordial black holes with interstellar antiprotons
}

\author{
James D. Wells ${ }^{1,2}$, Alexander Moiseev ${ }^{3}$, Jonathan F. Ormes ${ }^{3,4}$
}

\begin{abstract}
Interstellar antiproton fluxes can arise from dark matter annihilating or decaying into quarks or gluons that subsequently fragment into antiprotons. Evaporation of primordial black holes also can produce a significant antiproton cosmic-ray flux. Since the background of secondary antiprotons from spallation has an interstellar energy spectrum that peaks at $\sim 2 \mathrm{GeV}$ and falls rapidly for energies below this, low-energy measurements of cosmic antiprotons are useful in the search for exotic antiproton sources. However, measurement of the flux near the earth is challenged by significant uncertainties from the effects of the solar wind. We suggest evading this problem and more effectively probing dark-matter signals by placing an antiproton spectrometer aboard an interstellar probe currently under discussion. We address the experimental challenges of a light, low-power-consuming detector, and present an initial design of such an instrument. This experimental effort could significantly increase our ability to detect, and have confidence in, a signal of exotic, nonstandard antiproton sources. Furthermore, solar modulation effects in the heliosphere would be better quantified and understood by comparing results to inverse modulated data derived from existing balloon and space-based detectors near the earth.
\end{abstract}

\footnotetext{
${ }^{1}$ CERN, Theory Division, CH-1211 Geneva 23, Switzerland

${ }^{2}$ Stanford Linear Accelerator Center, Stanford University, Stanford, CA 94309

${ }^{3}$ NASA/Goddard Space Flight Center, Greenbelt, MD 20771

${ }^{4}$ This work was started while on leave at Stanford Linear Accelerator Center
} 


\section{Introduction}

Experimental and theoretical investigations of galaxy rotation, large-scale structure formation, big bang nucleosynthesis, and other observables appear to have converged on the necessity of dark matter (Trimble 1987; Sikivie 1995). Dark matter is non-luminous matter

which comprises a significant amount of the mass density of the universe. It may be mostly in the form of weakly interacting massive particles (WIMPs), and it may be due, at least in part, to primordial black holes (PBHs). It is not enough to hypothesize new stable particles and other interesting cosmological remnants. These objects must be found or excluded experimentally to make progress in our understanding of how the universe is put together. It is toward this goal that we write this paper.

There are numerous possible ways to find dark matter. If the WIMPs interact with ordinary matter then collisions of WIMPs with nuclei in the sun and earth could dissipate the WIMPs' energy enough to be captured. When they accrue in the sun and earth they will annihilate rapidly. The annihilation products will then fragment into neutrinos, some of which have high momentum directed toward earth-based neutrino telescopes. Current estimates in the supersymmetric case suggest that this is a difficult way to see dark matter unless there is an enhanced spin-dependent nuclear interaction (Jungman, Kamionkowski \& Griest 1996; Diehl et al. 1995).

One can search for dark matter also in a cryogenic detector (Cabrera 1998). With enough target material, it might be possible to see ambient WIMP collisions with cooled nuclei in the laboratory. The current estimate of the mass density of WIMPs in our solar system is $\rho \simeq 0.3 \mathrm{GeV} / \mathrm{cm}^{3}$, which means that there is only a handful of weak-scale mass WIMPs per cubic meter. Finding a signal in cryogenic detectors would be difficult unless there is a large coherent scalar interaction between the WIMP and the heavy nuclei in the cryogenic detector. Scalar interactions couple coherently to the nucleus, and so the counting rate is proportional to the mass of the nucleus not the mass of the constituent protons and neutrons. Therefore, larger mass nuclei are generally preferred for cryogenic detectors to take advantage of this possibility (Griest 1988).

Both the neutrino and cryogenic experimental programs rely on the dark matter interacting with ordinary matter. However, it is possible that the dark matter does not interact directly with ordinary matter. It is also possible that our local density is much smaller than the standard estimates indicate. In these circumstances, the above two experimental approaches may not find a signal for dark matter. Additional experimental methods are necessary to complete the search strategy for dark matter.

Annihilations and decays of dark matter in the galactic halo can yield ordinary particles 
even though the dark matter does not interact directly with them. This is true in the case of dark matter which carries no standard model quantum numbers. The annihilation or decay products are a sum over particles and antiparticles with zero net charge. Supersymmetry dark matter candidates generally have no quantum numbers, and fit in this category. This is also true of primordial black holes. Therefore, searches for annihilations or decays of dark matter into photons, positrons and antiprotons in cosmic rays are useful probes.

There are several sources of photons from dark matter. Annihilations into quarks and gluons which fragment into neutral pions which then decay into photons is one source. In the case of WIMP annihilation this gives a continuous spectrum of photons steeply falling with energy, $0 \lesssim E_{\gamma} \lesssim m_{\text {WIMP }}$. The standard photon background is also a steeply falling function of $E_{\gamma}$. This makes continuum photon signals difficult to resolve from background. However, one can use the measured photon background as a veto against all combinations of particle physics and astrophysics models which yield an unacceptably large photon flux. Another way to perhaps see dark matter is from interactions with electrons near the accretion disk of an active galaxy (Bloom \& Wells 1998). High energy photons emitted from an AGN at high angles may be indicative of non-standard interactions near the AGN. The non-uniqueness of the photon signal energy distribution and the expected small rate make this technique challenging as well. Yet another source of photons, and perhaps the most promising, is WIMP annihilation directly into two photons. Since the dark matter is not charged, this must proceed via a loop diagram. Since these loop diagrams are suppressed by factors of $\alpha / 4 \pi$ (where $\alpha=1 / 137$ is the QED coupling constant), the total rate for this observable is small. However, since the expected velocity of the WIMPs is non-relativistic $\left(\sim 10^{-3} c\right)$, the resulting photons are monochromatic. High photon energy resolution detectors may be able to see this type of signal, but the low rate is still a challenge (Bergström, Ullio, \& Buckley 1998; Bloom et al. 1998).

Positrons may also arise from the annihilation and decay products of dark matter. The largest source of positrons is from fragmentation of quarks into charged pions which then decay to positrons. The energy spectrum here is also continuous, and resolving signal positrons from background positrons is difficult. However, if the dark matter annihilates into a $W$ boson (or a top quark which then decays to a $W$ boson) then the positron from $W^{+} \rightarrow e^{+} \nu$ may have a non-trivial energy profile with distinctive bumps and peaks in the $e^{+} /\left(e^{+}+e^{-}\right)$spectrum (Turner \& Wilczek 1990; Kamionkowski \& Turner 1991; Diehl et al. 1995; Barwick et al. 1997 and 1998). However, the peakedness of the signal gets washed out somewhat when the parent particles of the positrons are boosted by a significant amount (i.e., $\left.m_{\text {WIMP }} \gg m_{W}\right)$. Furthermore, the signal is not present if a $W^{+}$is not present in the decay chain. The positron signal also suffers from QED energy loss effects which serve to broaden any positron peak when it is propagated over the galaxy on its way to the detector. 
Each of these modes of detecting dark matter has difficulties that are hard to control theoretically and experimentally. Here, we will be mainly interested in antiproton searches of dark matter. When dark matter annihilates or decays it can produce QCD jets which fragment into antiprotons. If enough antiprotons are produced in this process it is hoped that they will be detected above background. As will be discussed below, antiprotons are qualitatively different than continuum photons and positrons as probes of dark matter. The unique feature of antiproton experiments is the peaking behavior in the background antiproton energy distribution. The signal does not have this feature which makes separation of signal from background possible.

In the next section we review the standard sources of the antiproton cosmic ray component and discuss the challenges experiments face when trying to extract a signal of dark matter annihilations and/or decays. The challenges are significant for the experiments being performed near the earth, where antiprotons are battered by a hefty solar wind. We then discuss the experimental challenges of detecting low-energy antiprotons at the necessary sensitivities. The size, weight, and power consumption requirements for any apparatus bound for interstellar space is confronted, an initial design is presented, and sensitivities are estimated. We then make some concluding comments in the last section.

\section{Antiproton probes of dark matter and PBHs}

It was long ago acknowledged that one could potentially see dark matter by looking for an excess of antiprotons in cosmic rays from the annihilation or decay products of dark matter particles (Stecker, Rudaz, \& Walsh 1985; Rudaz \& Stecker 1988; Ellis et al. 1988; Jungman \& Kamionkowski 1994). This approach is unique since it is the background which has a non-trivial energy dependence, whereas the signal is a steeply falling function with increasing energy of the antiproton. The background antiprotons are produced by interactions of the high-energy proton cosmic rays with interstellar gas and dust (mostly other protons essentially at rest). To conserve baryon number $p p$ collisions must produce a final state with baryon number 2. The lowest multiplicity final state where this is possible, and where antiprotons are also created is $p p \rightarrow p \bar{p} p p$. To produce this final state, the incident proton must have kinetic energy $K>6 \mathrm{GeV}$ with respect to the at-rest proton target $\left(K \equiv E_{\bar{p}}-m_{\bar{p}}\right)$. At threshold in the center-of-mass frame, the $\bar{p}$ is at rest. Boosting back into the lab (or cosmic) frame, the $\bar{p}$ has a non-zero kinetic energy of about $\sim 2 \mathrm{GeV}$. To produce a $\bar{p}$ with kinetic energy any less than this requires a higher energy proton impinging on the at-rest proton such that the $\bar{p}$ in the center-of-mass frame has momentum in a direction opposite to the boost direction which takes the center-of-mass frame back 
to the lab frame. Since the primary proton flux is a steeply falling function of energy, the secondary antiproton flux must decrease for kinetic energies less than $\sim 2 \mathrm{GeV}$. In Fig. 1 the solid line is the background interstellar $\bar{p}$ flux from secondary processes, including $p p$ collisions and proton collisions with heavy nuclei (Simon, Molnar, \& Roesler 1998; See also, Stephens \& Golden 1987; Webber \& Potgieter 1989; Gaisser \& Schaefer 1992; Labrador \& Mewaldt 1997; Bottino et al. 1998).

Several attempts have been made to correlate theoretical models with the antiproton spectrum. One attractive theoretical framework is low energy supersymmetry which naturally allows for a stable lightest supersymmetric partner (LSP). In supersymmetric theories with gauge coupling unification and radiative electroweak symmetry breaking (Higgs mechanism derived radiatively), the relic abundance of the LSP is typically near closure density, making the LSP an excellent cold dark matter candidate (Drees \& Nojiri 1993; Kane et al. 1994; Diehl et al. 1995). More flexible approaches to supersymmetry also predict the LSP able to have a significant component of the cold dark matter of the universe (Wells 1998). The LSP in these cases is the lightest neutralino which carries no quantum numbers and can annihilate efficiently into standard model final states such as $b \bar{b}$. The fragmentation products of these final states include antiprotons, and predictions can be made for how the LSP annihilations change the antiproton energy spectrum from the expectations of secondary mechanisms.

The interstellar $\bar{p}$ flux from supersymmetry is represented (Diehl et al. 1995) by

$$
\Phi_{\bar{p}}^{\mathrm{IS}}(K)=\frac{(\sigma v)_{A B}}{4 \pi} \frac{\rho_{\mathrm{loc}}^{2}}{m_{\chi}^{2}}\left(v_{\bar{p}} \tau_{\bar{p}}\right) F_{\bar{p} / A B}\left(K, m_{\chi}\right)
$$

where $\rho_{\text {loc }} \simeq 0.3 \mathrm{GeV} / \mathrm{cm}^{3}, K$ is the kinetic energy of an antiproton, $v_{\bar{p}}$ is the velocity, and $\tau_{\bar{p}}$ is the containment time in the galaxy $\left(\sim 5 \times 10^{7} \mathrm{yrs}\right) . F_{\bar{p} / A B}\left(K, m_{\chi}\right)$ is the fragmentation function quantifying the multiplicity and energy distribution of antiprotons in

$$
\chi \chi \rightarrow A B \rightarrow \bar{p}+\cdots .
$$

Clearly, Eq. ili contains significant particle physics uncertainties in $m_{\chi}$ and the annihilation cross-section $(\sigma v)_{A B}$ from not knowing the underlying supersymmetry breaking parameters. However, for any given set of supersymmetric parameters these values are reliably computed. There is also some small and insignificant particle physics uncertainties in $F_{\bar{p} / A B}\left(K, m_{\chi}\right)$, but our knowledge of the remaining pieces of Eq. $i_{-1}^{5}$ are limited by astrophysics uncertainties. Our ignorance of many astrophysics parameters constitutes at least a factor of 10 uncertainty in the signal flux predictions by themselves.

The only energy range where the antiprotons from LSP annihilation are expected to be more copious than antiprotons from spallation is in the sub- $\mathrm{GeV}$ region. Unfortunately, 
this is precisely the area where solar modulation is most volatile. Solar modulation not only attenuates the flux of low energy protons and antiprotons, it also can dissipate some of the energy of these particles as they travel within its domain, which extends $\lesssim 60 \mathrm{AU}$ (Simpson 1989) radially from the sun. (For an illustration of solar wind effects on low energy protons and antiprotons, see Figs. 1 and 2 in Labrador \& Mewaldt 1997.) Therefore, excesses of antiprotons observed at these low energies may be hard to interpret as evidence for dark matter. For this reason, we will be interested in the flux of interstellar antiprotons in a small window of kinetic energy, $\Delta K$, below $1 \mathrm{GeV}$. The observable we want to measure is

$$
I_{\bar{p}}(\Delta K)=\int_{\Delta K} d K \Phi_{\bar{p}}^{\mathrm{IS}}(K) \epsilon(K)
$$

where $\epsilon(K)$ is the experimental acceptance efficiency of an antiproton with kinetic energy $K$. The units of $I_{\bar{p}}(\Delta K)$ are $\mathrm{cm}^{-2} \mathrm{sec}^{-1} \mathrm{sr}^{-1}$. In the next section we shall discuss the experimental apparatus to measure this with $\Delta K$ corresponding to $50 \mathrm{MeV}<K<200 \mathrm{MeV}$.

Another potential source for interstellar antiprotons is evaporation of Primordial Black Holes (PBHs) (Hawking 1974; Carr 1985; Page \& Hawking 1976; Kiraly et al. 1981; Turner 1982). These can be generated if significant density fluctuations arise in the early universe causing gravitational collapses in overdense regions (Hawking 1971; Carr 1985). Other ways to produce PBHs have been envisioned (Hawking et al. 1982; Hawking et al. 1989). Most of the mechanisms admit scale-invariant density perturbations which leads to a continuous spectrum of masses. The number density can be parametrized (Carr 1975) as

$$
\frac{d n}{d M}=\frac{(\beta-2) \Omega_{\mathrm{PBH}} \rho_{c}}{M_{H}^{2}}\left(\frac{M_{H}}{M}\right)^{\beta}
$$

where $\rho_{c}$ is the closure critical density of the universe, $\Omega_{\mathrm{PBH}}$ is the fraction of $\rho_{c}$ attributable to PBHs which have not yet evaporated, and $\beta$ is calculated from the equation of state when the PBHs were formed ( $\beta=2.5$ in the radiation-dominated era).

Standard Hawking evaporation calculations show that any PBH which has mass less than $M_{H} \simeq 10^{15}$ grams (the "Hawking mass") would have already decayed before our present time (Page 1976; MacGibbon \& Carr 1991; Halzen et al. 1991). The PBHs which are of most interest in seeing evaporation products are those black holes which are currently decaying, and so have mass near $M_{H}$. Thus, limits on primordial black hole density $\left(\Omega_{\mathrm{PBH}}\right)$ come from the number density of PBHs with $M \sim M_{H}$. Using the simplest scale-invariant density perturbation assumption which leads to Eq. ${ }_{-1}^{\bar{A}}$ one finds (MacGibbon \& Carr 1991; Halzen et al. 1991$)$ that $\Omega_{\mathrm{PBH}} \lesssim 10^{-8}$ from diffuse $\gamma$-ray observations. Roughly equivalent limits (Maki, Mitsui, \& Orito 1996; Mitsui, Maki, \& Orito 1996) can be found from currently available $\bar{p}$ measurements (Yoshimura et al. 1995; Mitchel et al. 1996; Moiseev et 
al. 1997; Matsunaga et al. 1998; Orito et al. 1998). An interstellar antiproton spectrometer would significantly increase these limits, and could allow for discovery.

One might think that such apparently extreme limits on $\Omega_{\mathrm{PBH}}$ seem to imply that PBHs are irrelevant to the structure and history of the universe. However, it should be noted, for one, that if the evaporated PBHs leave behind stable objects with mass above $m_{\mathrm{PL}}$, they could provide the cold dark matter of the universe even if $\Omega_{\mathrm{PBH}}<10^{-8}$ (MacGibbon 1987). Therefore, PBHs with mass density near the current limit is an interesting possibility with potentially dramatic dark matter considerations ${\stackrel{1}{\mathbf{a}_{1}}}_{\mathbf{r}}$

The $\bar{p}$ profile of PBH evaporation is slightly different than that of LSP annihilations and so a detailed $\bar{p}$ energy spectrum might be able to resolve the differences if hints of new physics beyond spallation appear to be required to explain data. However, like LSP annihilations, the large excesses of $\bar{p}$ 's are expected to be in the sub-GeV region. Therefore, the crushing effects of solar modulation muddy the experiments performed in the heliosphere near the earth.

The solution to these problems is to not be affected by solar modulation. Any experiment near the earth will necessarily be affected by the solar wind even at solar minimum (Perko 1987; Webber \& Potgieter 1989; Gaisser \& Schaefer 1992; Labrador \& Mewaldt 1997; Simon, Molnar, \& Roesler 1998). Therefore, to more effectively probe the antiproton spectrum for signs of exotic processes, such as LSP annihilation and PBH evaporation, it would help to put an antiproton spectrometer in interstellar space beyond the heliosphere, and therefore beyond the reach of significant solar modulation effects. Such a spectrometer could be one component of the payload in the recently discussed interstellar probe effort (Mewaldt et al. 1994).

In Fig. 1 we show the signal expected from the annihilations of the supersymmetric LSP, and also the decay of a PBH. The expectations have several astrophysical uncertainties. The most important uncertainty is the density profile of dark matter in our galaxy. The LSP curve shown in Fig. 1 is for the supersymmetric model of Bottino et al. 1998 with $m_{\mathrm{LSP}}=62 \mathrm{GeV}$, the LSP is mostly a gaugino-like neutralino with less than $2 \%$ Higgsino component, and $\Omega_{\mathrm{LSP}} h^{2}=0.11$. The assumed density profile is

$$
\rho_{\mathrm{LSP}}(r, z)=\rho_{\mathrm{loc}}\left\{\frac{a^{2}+r_{\odot}^{2}}{a^{2}+r^{2}+z^{2}}\right\}
$$

where $a=3.5 \mathrm{kpc}, r$ is the radial coordinate from galactic center, and $z$ is the coordinate

\footnotetext{
${ }^{a}$ This should not be confused with near critical mass density PBHs with a mass spectrum severely peaked at $\sim 1 M_{\odot}$ from non-scale-invariant perturbations (See, for example, Kawasaki, Sugiyama, \& Yanagida 1997).
} 
perpendicular to the galactic plane. Recent simulations (Navarro, Frenk, \& White 1996) indicate that the density profile may be much more cusping than the one given above. This cusping possibility introduces a systematic uncertainty in the astrophysics modeling of the dark-matter distribution. Although cusping would increase the antiproton signal, other uncertainties, such as clumping of dark matter in the halo, may decrease (or perhaps increase) the antiproton signature. The uncertainties in not knowing the precise particle physics model, and not knowing the precise astrophysical model of dark matter make it difficult to make definitive predictions of the signal antiproton flux. However, one does generically expect the flux of $\bar{p}$ 's below $1 \mathrm{GeV}$ due to near critical density supersymmetric dark matter to be comparable to secondary $\bar{p}$ 's.

In Fig. 1 we have also plotted the expected interstellar antiproton flux from a PBH evaporation calculation of Maki, Mitsui and Orito 1996. This is not the most optimistic prediction, nor is it the most pessimistic prediction of $\mathrm{PBH}$ evaporation. It is a prediction based on a reasonable set of assumptions and parameters. An upward turn or enhancement of the antiproton spectrum for kinetic energies below $1 \mathrm{GeV}$ would be an impressive clue that non-standard physics processes are occuring in the galaxy.

\section{Experimental discussion}

Very limited weight and power will be available for any experiment on board an interstellar probe. Those constraints dictate the design. We propose to use the annihilation signature of antiprotons that stop in a block of heavy material and release their entire rest mass energy ( $\sim 938 \mathrm{MeV})$. We base our design on a cube of heavy scintillator (BGO) with mass of the order of $1.5 \mathrm{~kg}$. That cube, $42 \mathrm{~g} / \mathrm{cm}^{2}$ thick, will stop antiprotons (and protons) of energy $\lesssim 250 \mathrm{MeV}$. A time-of-flight system (TOF) is used to select low energy, slow particles. Particles with energy less than $\sim 50 \mathrm{MeV}$ will not penetrate to the main crystal through the TOF counters, so this sets the lower energy limit.

The separation of antiprotons from protons is the most challenging aspect of the design. Any low energy $(<250 \mathrm{MeV})$ proton which would pass TOF selections cannot deposit more than its own kinetic energy in the block while stopping antiprotons release their rest mass energy through the annihilation. Antiprotons will be required to deposit more than $300 \mathrm{MeV}$. A proton can deposit comparable energy in this amount of material only through hadronic interaction; only protons with energy $\gtrsim 500 \mathrm{MeV}$ can efficiently do so. These higher energy protons will be separated from $<250 \mathrm{MeV}$ antiprotons by the TOF. As a conservative estimate we assume that all protons with energy above $500 \mathrm{MeV}$ have the potential to create a background of "antiproton like" events, and their integral flux in 
interstellar space would be $\sim 1 \mathrm{~cm}^{-2} \mathrm{~s}^{-1} \mathrm{sr}^{-1}$ (using local interstellar proton flux from Seo et al. 1987). An example antiproton flux from PBH evaporation is $\sim 10^{-6} \mathrm{~cm}^{-2} \mathrm{~s}^{-1} \mathrm{sr}^{-1}$ in the energy interval from 50 to $200 \mathrm{MeV}$ (Maki, Mitsui and Orito, 1996); the expected secondary antiproton flux is a factor of ten lower (see Fig.1). This gives us the requirement to have no more than one false "antiproton" from $10^{7}$ protons, an extremely challenging task.

The proposed instrument is shown in Fig. 2. We plan to use a $6 \mathrm{~cm} \times 6 \mathrm{~cm} \times 6 \mathrm{~cm}$ segmented piece of a heavy scintillator such as BGO to stop antiprotons and measure the energy of their annihilation. The choice of $\sim 250 \mathrm{MeV}$ as the highest antiproton energy to be detected is a compromise between desirable higher detectable energy and better time-of-flight separation. This energy brings us to the chosen dimensions of the crystal keeping in mind the necessity to minimize the detector's weight. Segmentation of this block is needed to remove high-Z low-energy nuclei, which deposit energy in a predictable, continuous pattern. It also helps to remove protons with energy between $300 \mathrm{MeV}$ (energy threshold in the crystal) and $500 \mathrm{MeV}$, which have large scattering in the crystal and consequently a longer path and larger energy deposition. Making the stopping block a $6 \times 6 \times 6$ array of $1 \mathrm{~cm}^{3}$ crystals will also allow us to form a crude image of the pattern of energy deposition. The TOF system, consisting of four $5 \mathrm{~mm}$ thick plastic scintillators spaced by $5 \mathrm{~cm}$, selects only low energy slow particles. The scintillator closest to the central $\mathrm{BGO}$ is $6 \mathrm{~cm} \times 6 \mathrm{~cm}$ and is divided in 2 strips, while the outer scintillator is larger, $12 \mathrm{~cm} \times 12 \mathrm{~cm}$ with 3 strips. Two scintillators in the middle of the TOF stack are $10 \mathrm{~cm} \times 10 \mathrm{~cm}$ and $8 \mathrm{~cm} \times 8 \mathrm{~cm}$. The trigger is the coincidence of all possible pair combinations (6) of time-of-flight detectors and must be above a time-duration threshold which corresponds to $\beta \simeq 0.7$. Moreover, all pulse heights from the 4 scintillators should be above a threshold which corresponds to the ionization loss for the appropriate velocity particle. This threshold will be about twice the mean energy loss of a minimum ionizing $\mathrm{Z}=1$ particle ( mip) to find slow particles and reject faster (lower $d E / d x$ ) ones. Finally, the trigger will require that the energy detected by the BGO crystal be above $\sim 300 \mathrm{MeV}$. A fast proton ( $\mathrm{mip}$ ) without an interaction loses $\sim 90 \mathrm{MeV}$ in the worst case of longest path traversed in the crystal. The time resolution of TOFs is assumed to be $50 \mathrm{ps}$ which is probably the best that can be presently achieved with scintillators of this size (Mitchell, private communication). All possible ways to reach and improve on this resolution should be explored. Currently we have simulation results that indicate a proton rejection power of $2 \times 10^{6}$ with a good hope of reaching the requirement by tightening selections and finetuning the instrument design. For example, additional rejection power can be obtained using more sophisticated on-board selection of events which corrects $d E / d x$ and transit time measurements from the scintillators for arrival direction. A major source of background in our simulations to date is due to timing fluctuations; chance coincidences will also play a 
role and must be explored carefully. We can also compare the pattern of energy deposition in the individual crystals to that expected from annihilations and interactions to achieve further background rejection. The efficiency, $\epsilon(K)$, of the antiproton acceptance after all the selections described above are applied is shown in Fig. 3. The energy resolution is $\sim 10 \%$ at $100 \mathrm{MeV}$, provided by TOF. The estimated dimensions of the instrument are $25 \mathrm{~cm} \times 20 \mathrm{~cm} \times 20 \mathrm{~cm}$ with $5 \mathrm{~kg}$ weight and $15 \mathrm{~W}$ of power. Experimental mass, the most critical parameter for a deep-space mission, is broken down into BGO crystal (2 kg), the TOF scintillators with frame and phototubes $(1.6 \mathrm{~kg})$, electronics $(1 \mathrm{~kg})$ and mechanical structure $(0.4 \mathrm{~kg})$. An on-board processor would analyze the data and reject most residual background. The data rate would be very low, $\sim 1000 \mathrm{bit} /$ day .

The estimated geometrical factor for this instrument would be $G \simeq 10 \mathrm{~cm}^{2} \mathrm{sr}$. The expected event rate would be 0.3 - 3 antiprotons per day between 50 and $200 \mathrm{MeV}$ energy. We can estimate the exposure time, $t_{e}$, needed to measure a signal to within $x \%$ statistical uncertainty:

$$
t_{e}=\frac{100}{I_{\bar{p}}(\Delta K)} \frac{1}{G}\left(\frac{10 \%}{x}\right)^{2} .
$$

Therefore, to obtain a $10 \%$ statistical precision measurement of the example $\bar{p}$ flux from PBH evaporation given in Fig. 1, where $I_{\bar{p}}(\Delta K) \simeq 4 \times 10^{-7} \mathrm{~s}^{-1} \mathrm{sr}^{-1} \mathrm{~cm}^{-2}$, one would require approximately $t_{e} \simeq 0.8 \mathrm{yr}$ of exposure time. Based on statistical inference only, both the LSP signal and the PBH signal in Fig. 1 would be detectable in approximately a year. Since exposure times for many years are possible for an interstellar mission, one could reduce the bandwidth and efficiency for more rejection power against protons.

\section{Conclusion}

The quest for dark matter has been a long one. Despite recognizing the potential existence of weakly interacting stable particles for many years, we have yet to find it or rule it out. The challenge is most explicitly made clear in a supersymmetric context, since a large class of models yield an LSP relic abundance near critical density (Wells 1998). Within these particular models, past and present dark-matter experiments are likely not to have a signal for LSP annihilations unless optimistic astrophysical parameters are assumed. Future detectors will cover much ground in the supersymmetric parameter space. As outlined in the beginning, each experiment has its advantages and weaknesses even within the specific supersymmetric framework. The advantage of the interstellar antiproton search is that it is more insensitive to the type of dark matter (WIMPs, LSPs or PBHs), and the signal is always expected to be continuous against a peaking background. Furthermore, 
measurements of the interstellar $\bar{p}$ flux would greatly aid the analysis of data from other experiments taking place near the earth (AMS, PAMELA, and others), since the interstellar $\bar{p}$ flux would be an extremely useful observable to compare with the inverse modulated near-earth data. Exploring how the spectrum changes as the detector leaves the heliosphere would be an added bonus to this project. This could further help our understanding of energy losses and flux modulations in the solar system. It is mainly for these reasons that we think an interstellar antiproton detector would be an excellent addition to the search for dark matter.

Acknowledgements: One of us, JFO, would like especially to thank the management at the Stanford Linear Accelerator Center for their hospitality during the period in which this work was initiated. 


\section{REFERENCES}

S.W. Barwick et al. (The HEAT Collaboration) 1997, ApJ, 482, L191.

S.W. Barwick et al. (The HEAT Collaboration) 1998, ApJ, 498, 789.

L. Bergström, P. Ullio, \& J.H. Buckley 1998, Astropart. Phys., 9, 137.

E. Bloom et al. (The GLAST Collaboration) 1998, SLAC Report 522.

E. Bloom \& J. Wells 1998, Phys. Rev. D, 57, 1299.

A. Bottino, F. Donato, N. Fornengo, \& P. Salati 1998, preprint astro-ph

B. Cabrera 1998, "Direct Detection WIMP searches below $100 \mathrm{~K}$ ", to be published in Proceedings 3rd International Symposium on Sources and Detection of Dark Matter in the Universe (DM 98), Marina del Rey, CA, 18-20 Feb 1998.

B.J. Carr 1975, ApJ, 201, 1.

B.J. Carr 1976, ApJ, 206, 8.

B.J. Carr 1985, in Observational and Theoretical Aspects of Relativistic Astrophysics and Cosmology, eds J.L. Sanz and L.J. Goicoechea, (Singapore: World Scientific, 1985).

E. Diehl, G.L. Kane, C. Kolda, \& J.D. Wells 1995, Phys. Rev. D, 52, 4223.

M. Drees \& M.M. Nojiri 1993, Phys. Rev. D, 47, 376.

J. Ellis et al. 1988, Phys. Lett. B, 214, 403.

T.K. Gaisser \& R.K. Schaefer 1992, ApJ, 394, 174.

K. Griest 1988, Phys. Rev. D, 38, 2357.

F. Halzen, E. Zas, J.H. MacGibbon \& T.C. Weekes 1991, Nature (London), 353, 807.

S.W. Hawking 1971, Mon. Not. R. Astr. Soc., 152, 75.

S.W. Hawking 1974, Nature (London), 248, 30.

S.W. Hawking et al. 1982, Phys. Rev. D, 26, 2681.

S.W. Hawking et al. 1989, Phys. Lett. B, 231, 237.

G. Jungman \& M. Kamionkowski 1994, Phys. Rev. D, 49, 2316.

G. Jungman, M. Kamionkowski \& K. Griest 1996, Phys. Rept., 267, 195.

M. Kamionkowski \& M.S. Turner 1991, Phys. Rev. D, 43, 1774.

G. Kane, C. Kolda, L. Roszkowski, \& J.D. Wells 1994, Phys. Rev. D, 49, 6173.

M. Kawasaki, N. Sugiyama, \& T. Yanagida 1997, preprint hep-ph [9710259.

P. Kiraly et al. 1981, Nature (London), 293, 120. 
A.W. Labrador \& R.A. Mewaldt 1997, ApJ, 480, 371.

J. MacGibbon 1987, Nature (London), 329, 308.

J.H. MacGibbon \& B.J. Carr 1991, ApJ, 371, 447.

K. Maki, T. Mitsui \& S. Orito 1996, Phys. Rev. Lett., 76, 3474.

H. Matsunaga et al. 1998, Phys. Rev. Lett, 81, 18.

R.A. Mewaldt, J. Kangas, S. Kerridge, \& M. Neugebauer 1994, Acta Astronautica, 35, Suppl. 267.

J.W. Mitchel et al. 1996, Phys. Rev. Lett., 76, 3057.

T. Mitsui, K. Maki \& S. Orito 1996, Phys. Lett. B, 389, 169.

A. Moiseev et al. 1997, ApJ, 474, 479.

J.F. Navarro, C.S. Frenk, \& S.D.M. White 1996, ApJ, 462, 563.

S. Orito et al. 1998, Proceedings of the International Conference on High Energy Physics (Vancouver, July 1998).

D.N. Page \& S.W. Hawking 1976, ApJ, 206, 1.

D.N. Page 1976, Phys. Rev. D, 13, 198.

J.S. Perko 1987, Astron. Astrophys., 184, 119.

S. Rudaz \& F.W. Stecker 1988, ApJ, 325, 16.

E.S. Seo et al. 1991, ApJ, 378, 763.

P. Sikivie 1995, Nucl. Phys. Proc. Suppl., 43, 90 43, 90 (1995).

M. Simon, A. Molnar, \& S. Roesler, ApJ, 499, 250.

J.A. Simpson 1989, Adv. Sp. Res., 9, (4)11.

F.W. Stecker, S. Rudaz \& T.F. Walsh 1985, Phys. Rev. Lett., 55, 2622.

S.A. Stephens \& R.L. Golden 1987, Space Science Rev., 46, 31.

V. Trimble 1987, Ann. Rev. Astron. Astrophys., 25, 525.

M.S. Turner 1982, Nature (London), 297, 82.

M.S. Turner \& F. Wilczek 1990, Phys. Rev. D, 42, 1001.

W.R. Webber \& M.S. Potgieter 1989, ApJ, 344, 779.

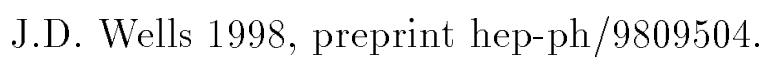

K. Yoshimura et al. 1995, Phys. Rev. Lett., 75, 3792. 


\section{Figure Captions}

Fig. 1.- The $\bar{p}$ interstellar flux, $\Phi_{\bar{p}}^{\mathrm{IS}}(K)$, versus kinetic energy from ordinary spallation processes (solid line — Simon, Molnar, \& Roesler 1998), from supersymmetric LSP annihilations for a particular set of supersymmertry breaking parameters with $m_{\chi}=62 \mathrm{GeV}$ (dashed line - Bottino et al. 1998), and primordial black hole evaporation (dash-dotted line - Maki, Mitsui, \& Orito 1996).

Fig. 2.- Diagram of the experimental design. See text for details.

Fig. 3.- The efficiency $\epsilon(K)$ for antiproton acceptance as a function of its kinetic energy after all the selections described in the text are applied. 


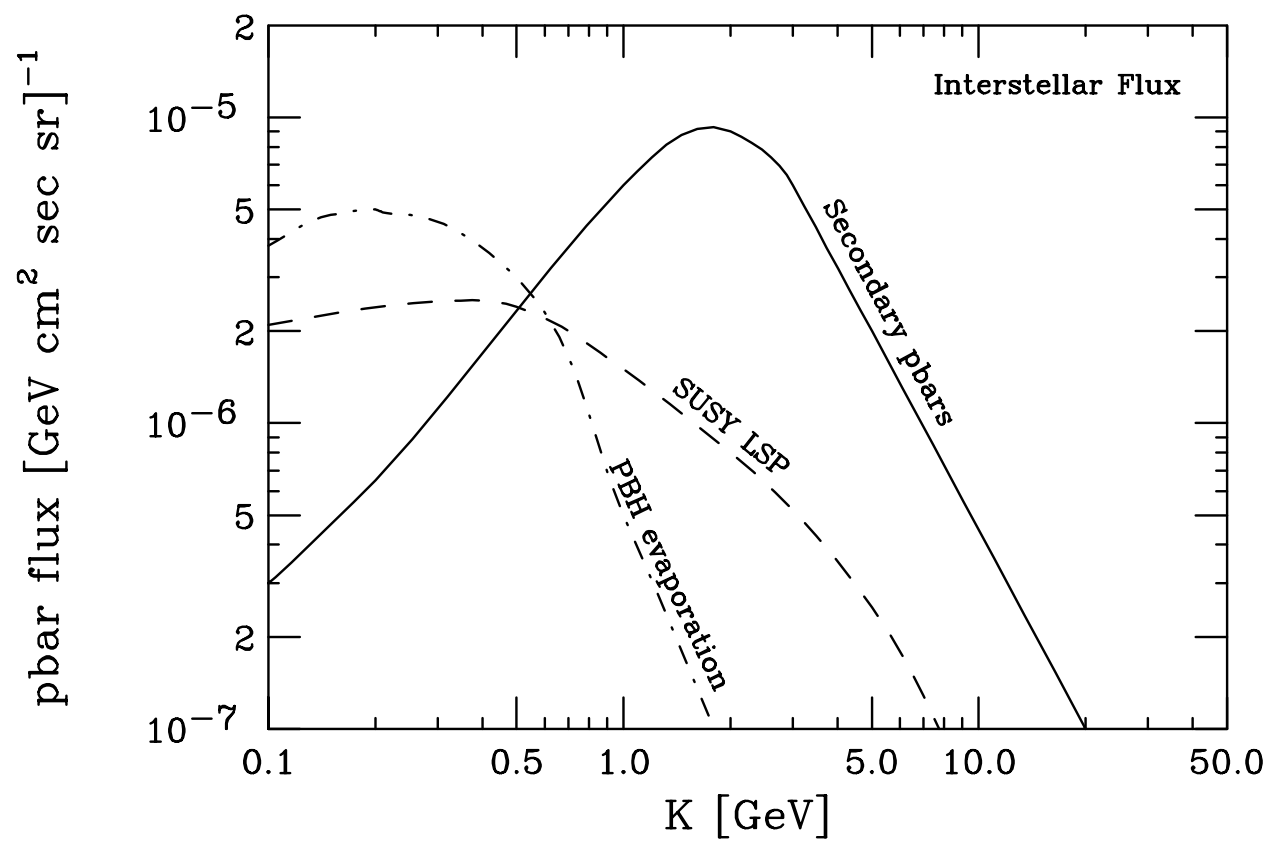

Fig. 1 


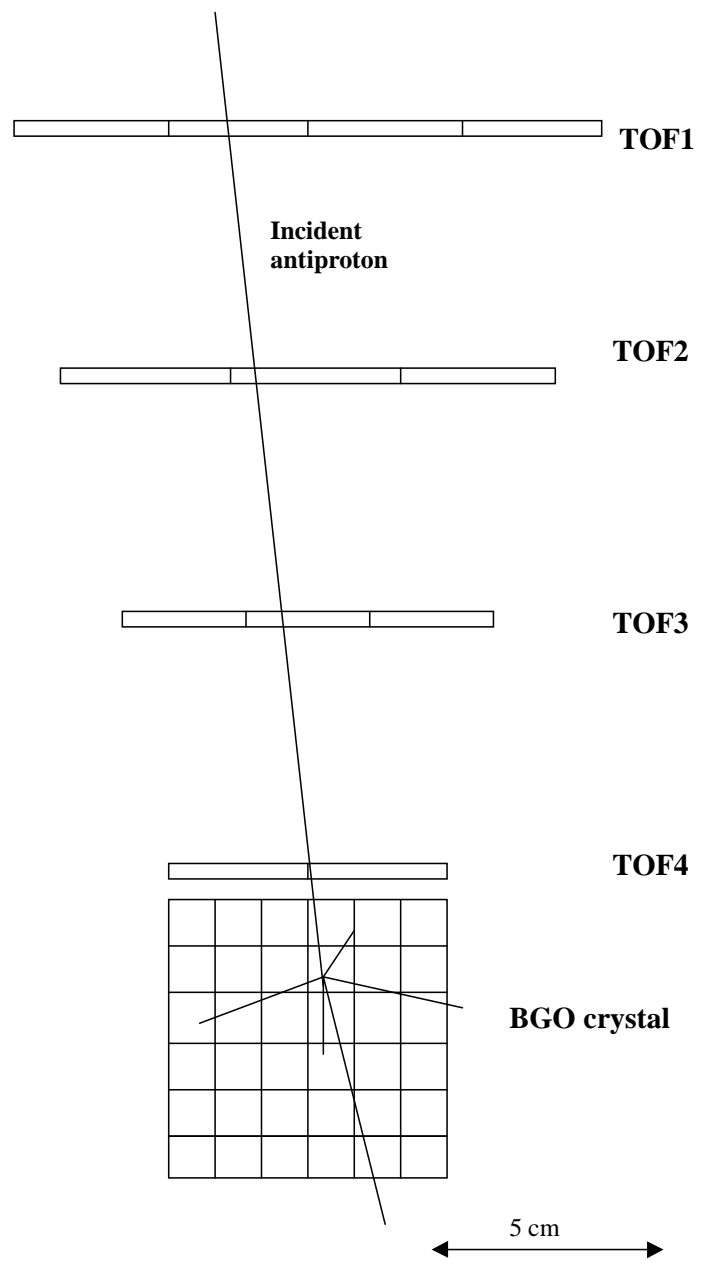

Fig. 2 


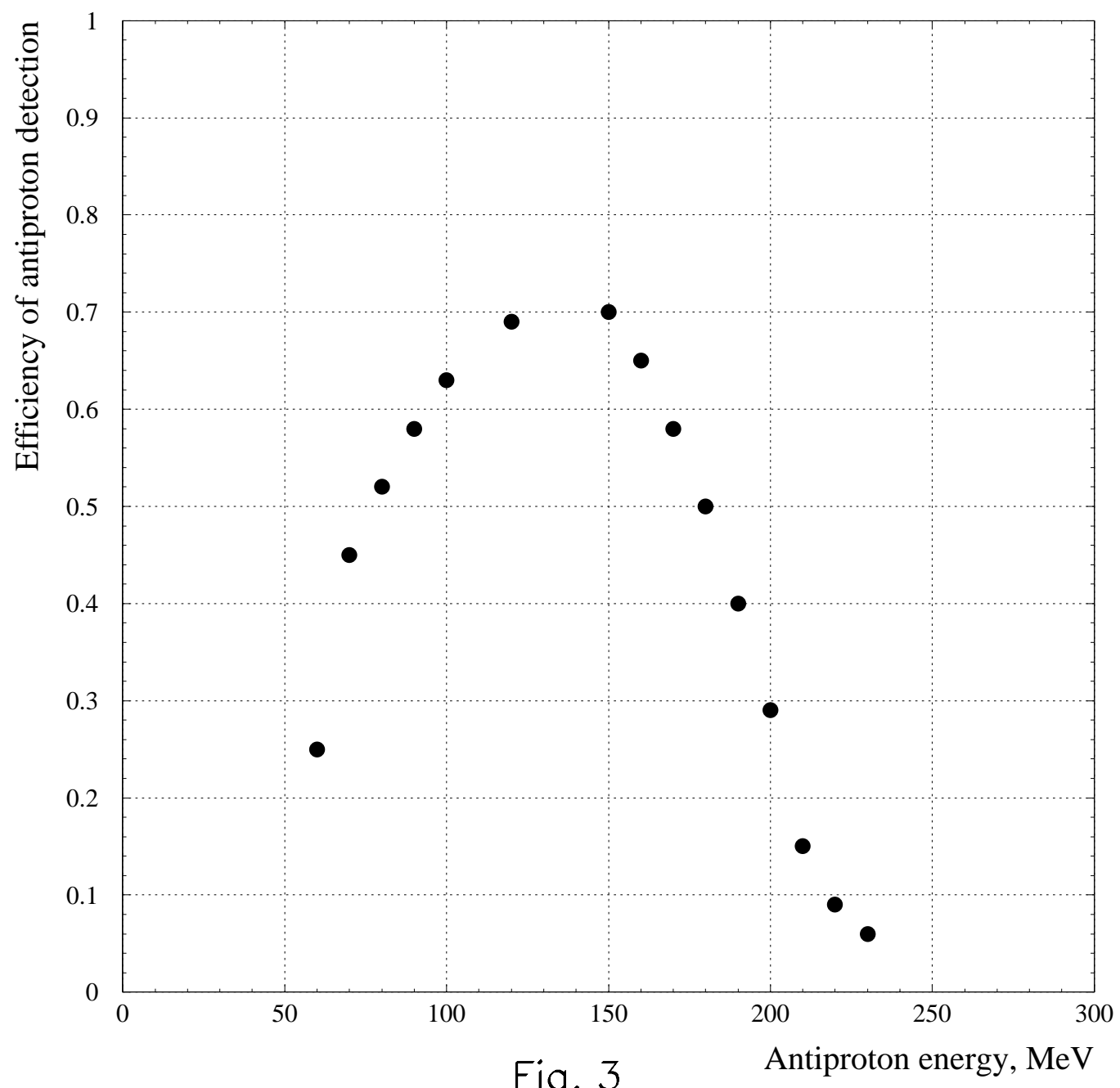

